\title{
Evaluation of HIV Status and its Spread in HIV Patients on Renal Replacement Therapy (RRT) and their Spouses
}

Aditya Agarwal ${ }^{*}$, Agarwal DK, Rao RR and Nalin Nag

Indraprastha Apollo Hospitals, New Delhi, India

\begin{abstract}
Background and methods: We studied the HIV status of patients on renal replacement therapy (RRT) and their spouses via a questionnaire. We selected 24 HIV positive patients who were either on maintenance hemodialysis $(n=14)$ or renal allograft recipients $(n=10)$. Patient's spouses $(20)$ were also included.

Results: Out of the 24 patients 21 were male (85.5\%) and 3 females (14.5\%). Only 20 patients had living spouses. Regarding probable source of infection; 20 (83.3\%) patients pointed towards multiple sexual contacts, while 1 each $(4.2 \%)$ stated blood transfusion and multiple needle pricks as the cause, while 2 were unsure of any cause (8.4\%). Out of 20 spouses, 18 were sexually active of which 8 (44.4\%) were found to be HIV Positive and all answered that they contracted the infection through sexual contact with their spouses. Among the 18 sexually active couples, 12 were using condoms, while 6 who were not using condoms were found to be HIV positive. Out of 8 HIV positive spouses, 2 were infected despite of using condoms. 15 patients out of 24 were chronic alcoholics $(62.5 \%)$. Out of 44 subjects (24 patients and 20 spouses) 40 opined that social worker counselling was the most effective way of preventing spread $(90.9 \%)$ followed by increasing awareness through media $(n=36,81.8 \%)$ while 30 subjects $(68.2 \%)$ stated that an addition to school curriculum would be helpful in preventing its spread whereas $28(63.6 \%)$ thought that counselling by the family physician could help in preventing the spread of the infection.
\end{abstract}

Conclusion: This study concludes that people with multiple sexual contacts, intravenous drug abusers and spouses of infected patients are at high risk of contracting HIV infection. Barrier contraception, though extremely effective, is not a full proof method of protection from the spread of HIV infection. Social workers have tremendous impact in increasing awareness of HIV and restricting its spread along with media, educational curriculum and physician counselling.

Keywords: Renal replacement therapy; HIV positive patients; End stage renal disease; Chronic kidney disease; Sexual transmission HIV; Spouse of HIV patients

\section{Introduction}

Human Immunodeficiency Virus (HIV) infection can cause chronic kidney disease (CKD) and can contribute significantly to the burden of patients requiring renal replacement therapy (RRT). The profile of patients with human immunodeficiency virus (HIV) infection has changed significantly since the mid-1990s, following the introduction of combined anti-retroviral therapy (cART). HIVassociated nephropathy (HIVAN) was previously the third most common cause of end-stage renal disease (ESRD) in African-American patients in the USA after hypertension and diabetes [1]. Since the advent of antiretroviral therapy (ART), HIVAN is now in 7th place as the cause of end-stage renal disease (ESRD) in African-American patients in the USA. In addition to HIVAN, the main causes of end stage renal disease include diabetic nephropathy, nephrosclerosis and nephrotoxicity [2]. However, the overall incidence of chronic kidney disease requiring renal replacement therapy (CKD-5) in these patients has not decreased. Nearly 4-7\% of HIV positive patients develop ESRD and it has become one of the most important causes of morbidity and mortality in HIV positive patients [3].

Introduction of combined antiretroviral therapy (cART) has increased the life expectancy of HIV positive patients who are on renal replacement therapy in the form of either maintenance hemodialysis or renal transplantation. Prolonged life expectancy in these patients also increases the chances of spread of HIV infection to their spouses $[4,5]$. We have carried this study in HIV positive patients who were on renal replacement therapy and their spouses, to evaluate HIV status and its spread in these patients and their spouses. We have also studied the level of awareness about the disease and methods of prevention among the subjects.

\section{Materials and Method}

This study included 24 HIV positive patients (tested through ELISA: Abbott Architect with CMI technology) who were on renal replacement therapy. Out of 24 patients, 20 patients had spouses. Majority of them were male $(21,85.5 \%)$ of black ethnicity (85.5\%). Out of 24 patients, 14 were on maintenance hemodialysis (58.3\%) while 10 were renal allograft recipients (41.6\%). All the patients were on cART. Most patients ( 16 out of 24 ) were on a combination of 2 NRTI (Nucleoside Reverse Transcriptase Inhibitor) and 1 NNRTI (NonNucleoside Reverse Transcriptase Inhibitor). HIV status of spouses was determined using ELISA method. Out of 20 spouses, 18 were female (90\%) and most of them were of black ethnicity (95\%). Hypertension (37.5\%) was the most common native kidney diagnosis followed by HIVAN (HIV associated Nephropathy), CIN (Chronic Interstitial Nephritis), DN (Diabetic Nephropathy) and the least common was CGN (Chronic Glomerulonephritis) (8.33\%). We interrogated these subjects via a multiple-choice questionnaire to know the causes of

*Corresponding author: Aditya Agarwal, MBBS, Indraprastha Apollo Hospitals, New Delhi, India, Tel: 009199998 00353; E-mail: dradityagarwal@gmail.com

Received January 23, 2017; Accepted February 02, 2017; Published February 09, 2017

Citation: Agarwal A, Agarwal DK, Rao RR, Nag N (2017) Evaluation of HIV Status and its Spread in HIV Patients on Renal Replacement Therapy (RRT) and their Spouses. J AIDS Clin Res 8: 662. doi: 10.4172/2155-6113.1000662

Copyright: ( 2017 Agarwal A, et al. This is an open-access article distributed unde the terms of the Creative Commons Attribution License, which permits unrestricted use, distribution, and reproduction in any medium, provided the original author and source are credited. 
spread and awareness among subjects. Opinions of these subjects were also taken regarding the ways of prevention of HIV spread. Prior approval was obtained from the ethical committee of the hospital for this study (Table 1).

\begin{tabular}{|c|c|}
\hline A. HIV Positive Patients on RRT & $\mathrm{N}=\mathbf{2 4}$ \\
\hline 1. Age (years), median (IQR) & $44.66(34,58)$ \\
\hline 2. Male, $\mathrm{N}(\%)$ & $21(85.5 \%)$ \\
\hline 3. Black ethnicity, $N$ (\%) & $21(85.5 \%)$ \\
\hline 4. Time since HIV diagnosis (year), median (IQR) & $3.58(2,8)$ \\
\hline \multicolumn{2}{|l|}{ 5. Viral hepatitis, $\mathrm{N}(\%)$} \\
\hline HBsAg positive & $0(0 \%)$ \\
\hline HCV Ab/HCV RNA positive & $3(8 \%)$ \\
\hline \multicolumn{2}{|l|}{ 6. Class of drugs in cART regimen, $\mathrm{N}(\%)$} \\
\hline a. $2 \mathrm{NRTI}$ and $1 \mathrm{NNRTI}$ & $16(66.6 \%)$ \\
\hline b. 2 NRTI and $1 \mathrm{PI}$ & $4(16.6 \%)$ \\
\hline c. 1 NRTI, 1 NNRTI, 1 PI & $2(8.3 \%)$ \\
\hline d. 2 NRTI and 1 Integrase inhibitor & $2(8.3 \%)$ \\
\hline \multicolumn{2}{|l|}{ 7. RRT N (\%) } \\
\hline Hemodialysis & $14(58.3 \%)$ \\
\hline Peritoneal dialysis & $0(0 \%)$ \\
\hline RT & $10(41.6 \%)$ \\
\hline \multicolumn{2}{|l|}{ 11. Native kidney disease, $\mathrm{N}(\%)$} \\
\hline a. HIVAN & $8(33.3 \%)$ \\
\hline b. CGN & $2(8.33 \%)$ \\
\hline c. CIN & $2(8.33 \%)$ \\
\hline d. DN & $3(12.5 \%)$ \\
\hline e. HTN & $9(37.5 \%)$ \\
\hline B. Spouses of HIV Positive Patients on RRT & $\mathrm{N}=\mathbf{2 0}$ \\
\hline 1. Age (years), Median (IQR) & $39.7(30,52)$ \\
\hline 2. Female $\mathbf{N}(\%)$ & $18(90 \%)$ \\
\hline 3. Black, Ethnicity N (\%) & $19(95 \%)$ \\
\hline
\end{tabular}

RRT: Renal Replacement Therapy; RT: Renal Transplant; HIV: Human Immunodeficiency Virus; HBsAg: Hepatitis B Surface Antigen; NRTI: Nucleoside Reverse Transcriptase Inhibitor; HCV: Ab-Hepatitis C Antibody; cART: Combination Antiretroviral Therapy; NNRTI: Non- Nucleoside Reverse Transcriptase Inhibitor PI: Protease Inhibitor; HIVAN: HIV Associated Nephropathy; CGN: Chronic Glomerulonephritis; CIN: Chronic Interstitial Nephritis; DN: Diabetic Nephropathy; HTN: Hypertension

Table 1: Baseline characteristics of patients and their spouses.

\section{Results}

24 HIV positive CKD V patients were included in this study. All patients were either on dialysis support in the form of hemodialysis or had received renal transplantation. Of the 24 patients, 20 had living spouses. Among the 20 spouses, 8 (40\%) were found to be HIV positive.

\section{Questionnaire}

\section{Probable mode of spread}

Out of the $24 \mathrm{HIV}$ positive patients, 20 (83.3\%) stated they had multiple sexual contacts and thus contracted HIV, while one patient each stated that they had contracted the virus through needle prick and Blood Transfusion ( $4.2 \%$ each), while 2 were unsure about the mode of spread (8.4\%).

\section{Chronic alcoholism}

Of these 24 patients on RRT, 15 patients $(62.5 \%)$ answered that they were chronic alcoholics. Although none of these patients exhibited clinical signs of Chronic liver disease (Figure 1).

\section{Sexually active}

Out of the 20 spouses, 18 stated that they were sexually active. Of these, 8 spouses were found to be HIV positive and $10 \mathrm{HIV}$ negative.

\section{Use of contraception}

Among the 18 sexually active couples, 12 used male barrier contraception consistently, out of which 2 were found to be HIV positive (16.6\%).

Whereas all 6 spouses who were sexually active without practicing barrier contraception in the form of condoms were HIV positive (100\%) (Figure 2).

\section{Ways/methods of prevention}

Out of 44 subjects ( 24 patients and 20 spouses), 40 opined that social worker visits with counselling were effective in preventing spread of the disease $(90.9 \%)$. Of the 44 subjects, 36 stated that newspaper, television and social media were good medium for the same $(81.8 \%)$. Whereas 30 favored teaching about HIV as being effective at schools
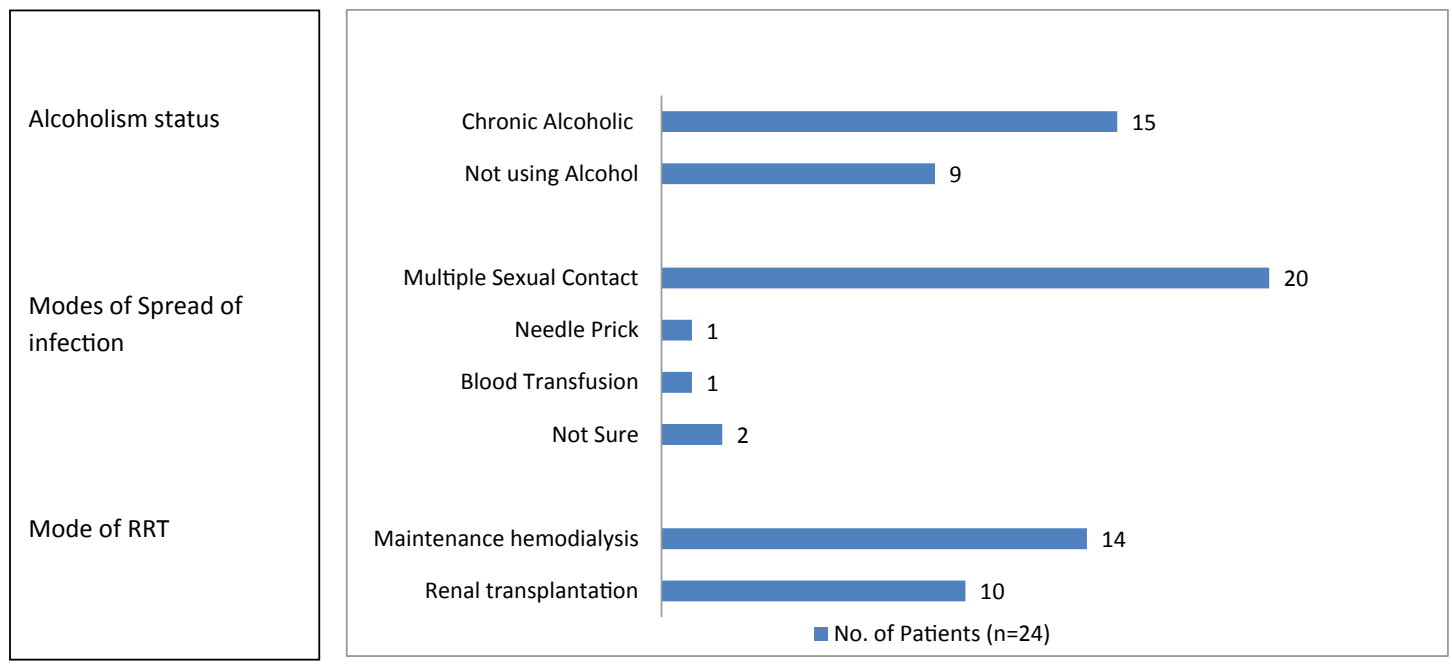

Figure 1: Showing modes of RRT,modes of spread of HIV infection and prevalence of alcoholism. 


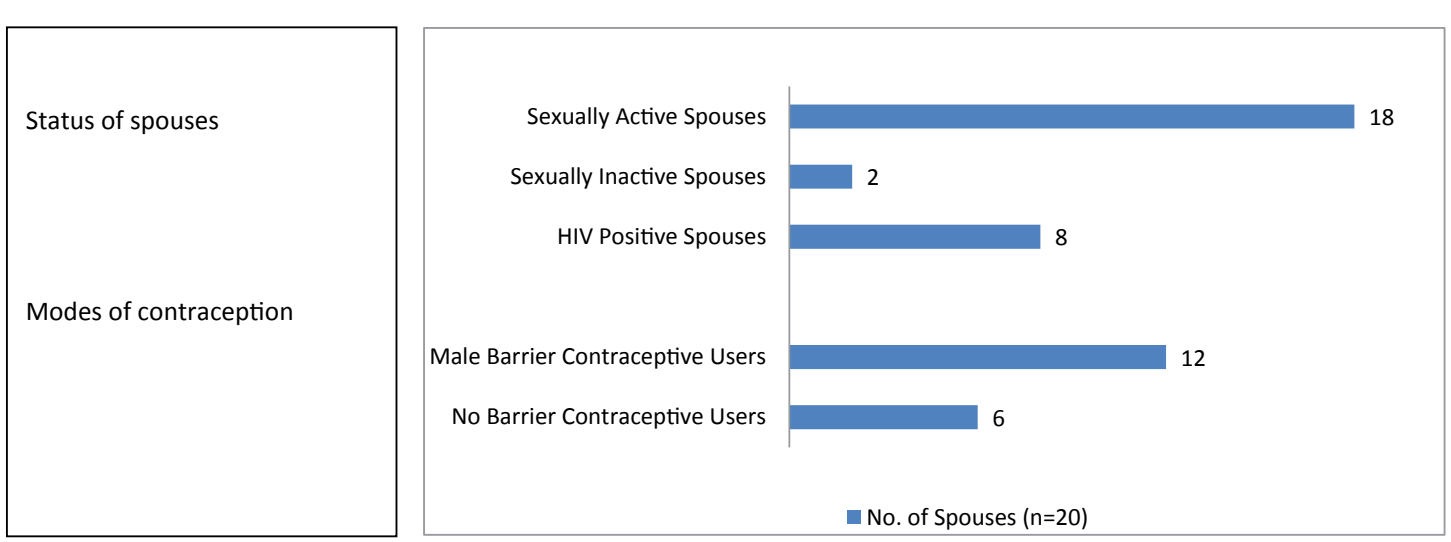

Figure 2: Showing modes of contraception and HIV positivity among spouses.

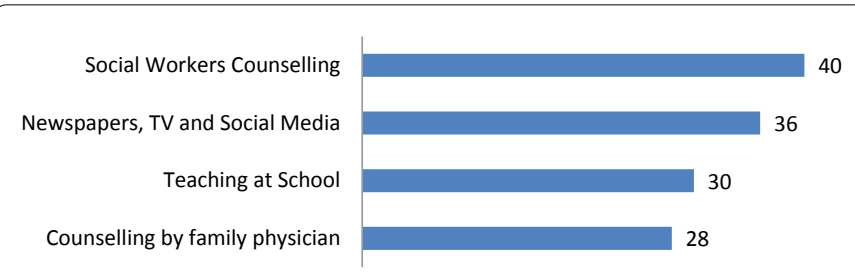

No. of Subjects Patients and Spouses $(\mathrm{n}=44)$

Figure 3: Showing methods of prevention of spread of HIV infection.

(68.2\%). Family physician counselling would be effective for increasing awareness and preventing the spread of the disease As per to 28 subjects $(63.6 \%)$ (Figure 3 ).

\section{Discussion}

This study was aimed at evaluation of HIV status and its spread in HIV patients on RRT and their spouses. In our study $85.5 \%$ were male and $14.5 \%$ female. Even the national (Indian) and worldwide data suggests a disparity in the prevalence of HIV positivity between males and females. This may be multifactorial and therefore requires further investigation. One factor may be that overall; males receive more attention towards their health in comparison to females in our society.

Multiple patients answered that they caught the infection due to multiple sexual contacts. Globally known main modes of transmission of HIV infection are through sexual contact, needle prick injury, blood transfusion. HIV is spread through certain body fluids from an infected individual. These fluids include blood, semen, pre-seminal fluids, rectal fluids, vaginal fluids, and breast milk. As per Modes of Transmission model by UNAIDS (Joint United nations program on HIV/AIDS), most common mode of transmission is through sexual contact (31.87-33.26\%), followed by Intravenous drug use $(0.77 \%)$, Needle prick injury $(0.09 \%)$ and Blood transfusion (0.09\%) [6]. Present study also concludes that the most common mode of spread was through sexual contact.

Among the 20 patients with spouses, 8 spouses were found to be HIV positive. As per this study, there is increased HIV positivity in spouses of HIV patients. Of the 18 couples who were sexually active, 12 used male barrier contraception consistently and only 2 spouses of these patients were HIV positive. While the remaining 6 spouses who did not use barrier contraception were HIV positive suggesting sexual mode of spread is of particular importance leaving spouses of HIV patients at high risk for transmission of HIV infection.
Male condoms are an extremely effective means of HIV, STD and pregnancy prevention. The effectiveness of condom is limited by user failure rather than product failure. Users may fail to either put on a condom before genital contact or fail to completely unroll the condom. In addition, some people fail to use a condom with every act of sexual intercourse. Some don't use condoms because condoms reduce sexual sensation. Other feel that condoms are a barrier to intimacy [6].

Above may be the reasons of our observations that 2 spouses were infected in despite barrier contraceptive use. It means that barrier contraceptives in the form of male condoms, though extremely effective, are not full proof method of protection from the spread of HIV infection.

HIV prevention programmes are interventions that aim to halt the transmission of HIV. Initially, HIV prevention programs focused primarily on preventing the sexual transmission of HIV through behavior change. For a number of years, the ABC approach - "Abstinence, Be faithful, Use a Condom" - was used in response to the growing epidemic in sub-Saharan Africa. However, by the mid2000s, it became evident that effective HIV prevention needs to consider underlying socio-cultural, economic, political, legal and other contextual factors. As the complex nature of the global HIV epidemic has become clear, forms of 'combination prevention' have largely replaced ABC-type approaches [6]. In this study, most of the subjects opined that social workers along with social media may be key in the prevention of HIV spread.

\section{Conclusion}

HIV infection can cause CKD and HIV infection can also be acquired during renal replacement therapy. It increases both mortality and morbidity in CKD patients. Hence, awareness of HIV infection and prevention are necessary to decrease disease burden. Thus, this study concludes that multiple sexual contacts, intravenous drug use are main mode of transmission of HIV infection. Spouses of HIV positive patients on RRT are at high risk of contracting the infection. Barrier contraceptives are very effective but do not provide $100 \%$ protection. Spouses of CKD 5 patients on RRT are at higher risk for HIV infections. Social workers have tremendous impact on increasing the awareness about HIV and restricting its spread; along with media, inclusion in educational curriculum and physician's counselling. Further such studies with a higher sample size, more in depth questionnaire and selection of patients in different settings (for example hospitals, Out Patient Department, ART clinics, etc.) will further shed light on this issue. 
Citation: Agarwal A, Agarwal DK, Rao RR, Nag N (2017) Evaluation of HIV Status and its Spread in HIV Patients on Renal Replacement Therapy (RRT) and their Spouses. J AIDS Clin Res 8: 662. doi: 10.4172/2155-6113.1000662

\section{Acknowledgement}

We acknowledge the help of the DNB (Diploma of National Board) residents, nursing staff and OPD (Out Patient Department) staff of our institute for the collection and management of the data used in this study.

\section{References}

1. Ahuja TS, Collinge N, Grady J, Khan S (2003) Is dialysis modality a factor in survival of patients with ESRD and HIV-associated nephropathy? Am J Kidney Dis 41: 1060-1064

2. Mallipattu SK, Salem F, Wyatt CM (2014) The changing epidemiology of HIV-related chronic kidney disease in the era of antiretroviral therapy. Kidney Int 6: 259-265.
3. Rosenthal E, Poiree M, Pradier C, Perronne C, Salmon-Ceron D, et al. (2003) Mortality due to hepatitis C-related liver disease in HIV-infected patients in France (Mortavic 2001 study). AIDS 17: 1803-1809

4. Ahuja TS, Grady J, Khan S (2002) Changing trends in the survival of dialysis patients with human immunodeficiency virus in the United States. J Am SocNephrol 13: 1889-1893

5. Bhagani S, Sweny P, Brook G (2006) Guidelines for kidney transplantation in patients with HIV disease. HIV Med 7: 133-139.

6. UNAIDS (2010) Combination HIV prevention: Tailoring and coordinating biomedical, behavioural and structural strategies to reduce new HIV infections. 